\title{
Conception of the Theorem of Light and Heat
}

\author{
Guoyi Zheng \\ Baihe County Cultural Center, Ankang 725899, Shaanxi, China
}

\begin{abstract}
Light is physical. Light is the state of light particles moving in a straight line at a uniform speed at the speed of light (the maximum speed of light is $29.9792458 \mathrm{~km} / \mathrm{s}$ ). A light particle is a combination of cube-shaped particles (non-atomic structure) made up of four protons and four electrons. Its mass is (proton mass +electron mass) $\times 4$. Its protons and electrons are relatively static, and its overall positive and negative charges are balanced, but it has positive and negative polarities with multi-dimensional shapes, and electromagnetic phenomena will be generated in high-speed rotation. Light color is the vibration state of light particles in the process of uniform linear motion. The size of its jitter degree forms various light colors. Light particles are produced when atoms fission. Any gaseous atom or molecule (including mass particles) can be excited to the speed of light to emit visible light or rays. The greater the atomic weight or molecular weight of the gaseous state, the greater its light energy. Light particles are the only source of heat that carry heat and transfer heat in substances such as air and generate heat.
\end{abstract}

Keywords: Light, Light particle structure, Heat.

\section{The theorem of light}

\subsection{The Nature of Light and its Theorems}

Light is material. Light can be blocked by opaque objects, which proves the materiality of light and proves that light is not an electromagnetic wave. Light is made up of light particles. Light is the state of light particles moving in a straight line at a uniform speed at the speed of light (the maximum speed of light is $29.9792458 \mathrm{~km} / \mathrm{s}$ ). Any mass of matter can become light as long as it reaches the speed of light.

(1) A light particle is a cube-shaped particle combination (non-atomic structure) composed of four protons and four electrons. Its mass is (proton mass + electron mass) $\times 4$. Its protons and electrons are relatively static, and its overall positive and negative charges are balanced, but it has positive and negative polarities with multi-dimensional shapes, and electromagnetic phenomena will be generated in high-speed rotation. This electromagnetic phenomenon is a kind of electromagnetic induction, not the entity of light particles. It is like the electromagnetic shadow of light particles, which is called the electromagnetic shadow phenomenon of light particles. (Figure 1).
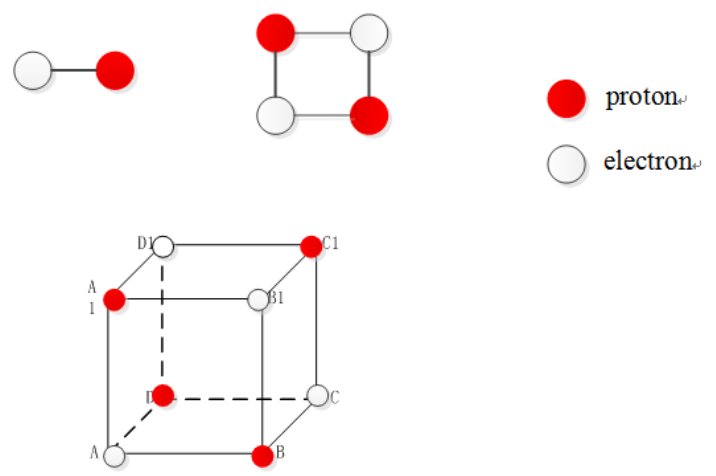

Figure 1: Optical particle structure

(2) The light particle has the inertia of maintaining a uniform linear motion forever, and the speed of its single rotation uniform linear motion in a vacuum is an original constant of $29.9792458 \mathrm{~km} / \mathrm{s}$, which is called the light speed constant.
(3) When a light particle moves in a uniform curve (including jitter (irregular vibration), vibration, fluctuation, and various circular motions), its linear velocity is always equal to the constant speed of light. At the same time, the relative linear velocity is formed.

(4) Light particles carry energy. When light particles do reflection motion and various field force motions, their energy is conserved, which is called light constant. The light constant is the energy and basis for light particles to participate in various particle motions. The magnitude of the light constant is called the light constant (represented by Q), that is, the light constant $\mathrm{Q}=$ the mass of the light particle $\times$ the square of the light speed constant.

(5) Under the action of the field force, the light particle changes its motion state and forms various superimposed composite motions, and its energy will not be lost.

(6) In the process of uniform linear motion, when the light particle hits the smooth material plane or the uniform field force plane, physical reflection occurs and does not consume energy.

(7) When the light particle collides with other particles, its energy is reduced or increased, the linear velocity is changed, or attenuated or upgraded to other light colors and rays.

(8) Light color is the vibration state of light particles in the process of uniform linear motion. The size of its jitter degree forms various light colors. White light (sunlight, etc.) is a state of high-speed rotation of light particles that move in a straight line at a uniform speed of $300,000 \mathrm{~km} / \mathrm{s}$. Other light colors are the state of light particles in a relatively uniform linear motion at various vibration frequencies. Red light, orange light, yellow light, green light, blue light, indigo light, and violet light are formed in sequence.

(9) Under the action of positive and negative electrodes, light particles can be connected to each other to form chains or streamlines, or large-area, large-volume and other geometric shapes, which are called photon arrays. After the light particles are joined together, they will no longer continue to rotate, and will be transformed into array vibrations or waves 
or relatively linear propagation. The propagation forms of light particles are divided into single-rotation uniform straight-line propagation, chain streamlined straight-line propagation, streamlined wave propagation, large-area straight-line propagation, and large-volume straight-line propagation.

(10) Sunlight is composed of parallel rays and intersecting rays. Outside the sun light source, the sun rays passing through any direction are always parallel rays; the sun rays passing through any point are cross rays. When the crossed sun rays pass through the prism, the light color and spectrum are formed after repeated and orderly reflection of the light channel.

(11) Light particles are produced when atoms fission. Any gaseous atom or molecule (including mass particles) can be excited to the speed of light to emit visible light or rays. The greater the atomic weight or molecular weight of the gaseous state, the greater its light energy. The energy of light can be increased by synthesizing atomic isotopes or large molecular weights (including compound molecules).

The number of light particles contained in an atom (called the atomic light number $\mathrm{n}$ ) is:

Atomic light number $\mathrm{n}=$ atomic proton number (or electron number $) \div 4$

The light particle energy contained in an atom (called atomic light energy e) is:

Atomic light energy e $=$ atomic light number $\mathrm{n} \times$ light constant Q

The light ray (invisible light) energy contained in an atom (called atomic light ray energy $r$ ) is:

Atomic light ray energy $r=$ atomic light number $n \times$ light particle mass $\times$ ray velocity squared

(12) After the fission of light particles, they are converted into proton rays or rays and electron rays or rays.

(13) Although light particles are non-atomic structures, their overall properties have properties similar to those of atoms, and can be defined as a new element (ie, light element). Light particles have similar chemical properties to hydrogen atoms, and can directly participate in biological and chemical reactions to generate hydrogen or hydride. Electrolysis of sunlight may generate hydrogen or other elements, revealing the origin of matter in the universe.

\subsection{Metric Values}

Light particle mass $=($ proton mass + electron mass $) \times 4$

Light speed constant $=29.9792458 \mathrm{~km} / \mathrm{s}$

Light constant $\mathrm{Q}=$ light particle mass $\times$ light velocity constant squared
Atomic light number $\mathrm{n}=$ atomic proton number (or electron number $) \div 4$

Atomic light ray energy $r=$ atomic light number $n \times$ light particle mass $\times$ ray velocity squared

Light energy after light speed $\mathrm{E}=$ light particle matter mass $\mathrm{m}$ $\times$ light speed square $\mathrm{C}^{2} \times$ time $\mathrm{t}$

Or: light energy after light speed $\mathrm{E}=$ light particle material mass $\mathrm{m} \times$ light speed square $\mathrm{C} \times$ light path $\mathrm{S}$

Light energy after light speed $\mathrm{E}=$ light particle momentum $\mathrm{D}$ $\times$ optical path $S$

The energy quality of light $\mathrm{M}=$ light energy after the speed of light $\mathrm{E} \div$ the square of the speed of light $\mathrm{C}^{2}=$ light particle matter mass $\mathrm{m} \times$ time $\mathrm{t}$

Variable speed light energy formula: variable speed light energy $\mathrm{E}=$ light particle mass $\mathrm{m} \times$ instantaneous light speed square $\div 2$

The light energy $\mathrm{E}=$ the mass of the light particle material $\mathrm{m} \times$ the speed of light square $\mathrm{C}^{2} \times$ the new field time $\mathrm{t} \times$ the new field intensity $\div$ the original field intensity

Total light energy $\mathrm{E}=$ light particle material mass $\mathrm{m} \times$ light speed square $\mathrm{C}^{2} \times$ (original field time + new field time $\times$ new field intensity $\div$ original field intensity)

\subsection{Basic Definition Explanation}

\subsubsection{Optical particle structure process}

A large number of protons and electrons are released during the fission of matter atoms. Protons have a positive charge and electrons have a negative charge. When a proton and an electron are attracted, a binary conjoined body is formed, and this conjoined body has positive and negative polarities. When two binary conjoined bodies are attracted to each other, a quaternary plane conjoined body is formed. When two quaternary planes are joined together, an eight-element cube is formed, and a light particle is formed.

\subsubsection{Electromagnetic shadow waves}

Light particles have positive and negative polarities in multi-dimensional forms, and electromagnetic phenomena can be generated during high-speed rotation. This electromagnetic phenomenon is a kind of electromagnetic induction, not the entity of light particles. It is like the electromagnetic shadow of light particles, which is called the electromagnetic shadow phenomenon of light particles. The electromagnetic wave generated by this electromagnetic shadow phenomenon can accurately describe the energy state and motion state of light particles, and can also be loaded with other various field force waves, becoming the public carrier and propagation medium of various field force waves.

\subsubsection{Conception and inference of optical channel}


Light particles can pass through photoconductor substances in a motion state. A light channel is a hypothetical light particle vacuum geometry channel. It is a hollow cuboid molecular gap channel with a square (or regular polygon) cross-section, and its inner four walls are all reflective planes. Light particles can pass straight through the vertical section, or reflectively pass through the internal light-returning plane. Light channels may be ubiquitous in light-conducting substances (eg, water, glass, etc.). See Figure 2.

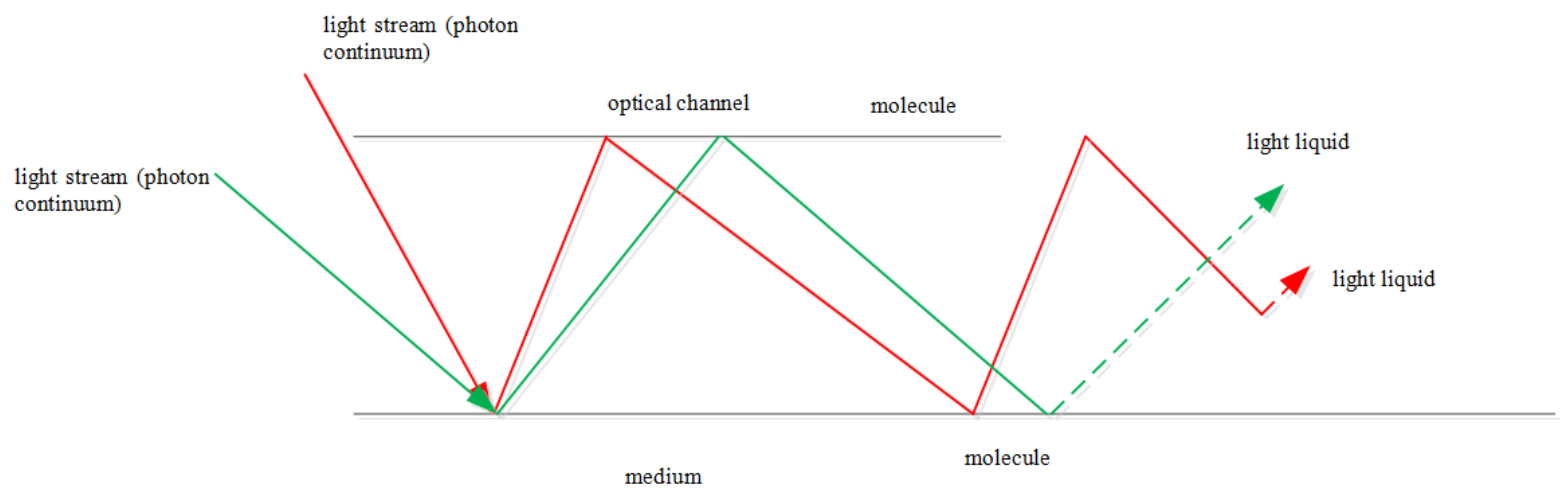

Figure 2: Principle of optical channel

When the light particle chain (white light such as sunlight) moving in a straight line at a uniform speed reflectively passes through the optical channel, it is repeatedly and orderly reflected, forming a wave-like linear propagation, and its frequency is determined by the incident angle, resulting in wavelength and frequency, thus forming various Light colors and rays (for example: red, orange, yellow, green, blue, indigo, violet, infrared ultraviolet, etc.). In this sense, it is inferred that other light colors are formed by the change of the motion state of white light, rather than white light containing these light colors, so white light is not composite light.

Therefore, light color is the state when light particles move at the speed of light in a certain form and value, and their own shaking state forms light color. The size of its jitter degree forms various light colors. Each light color has a fixed value of its own motion, followed by red light, orange light, yellow light, green light, blue light, indigo light, violet light (for example, sunlight produces spectral phenomena through a prism).

To sum up, when the light particles move in the medium, they will be simultaneously affected by the reflection surface of the micro-material inside the medium and the uniform field force, and the motion state will be changed. When light particles enter different media, under the action of the medium's gravitational force, they will be angularly deflected along the direction of their linear motion, resulting in refraction. When the light particle passes through the critical surface and the medium vertically, its motion direction does not change.

\subsubsection{Uniform field force reflection plane}

The reflection plane of light particles is divided into the material plane and the uniform field force plane. The uniform field force plane is a certain space geometric section in the uniform field force, which has all the space time, space position and space angle, which makes the light particles produce physical reflection.

\subsubsection{Relative linear motion}

Relative linear motion refers to the overall uniform linear motion state (such as fluctuation, spiral motion, etc.) exhibited by light particles when they perform various curvilinear motions, and generates a corresponding linear velocity, which is called relative linear velocity. When its velocity is less than at the speed of visible light, it cannot be seen.

The difference between the linear velocity constant of the light particle and the relative linear velocity shows the linear velocity occupied by a certain kind of curved motion made by the light particle. When a light particle moving in a straight line at a uniform speed makes a curved motion (such as a wave) under the action of a field force, its linear velocity will be folded or elongated, and its linear velocity value will not change, it is a constant. Its optical path (the actual length traveled by light particles) is also a constant.

\subsubsection{The sun's horizontal intersection duality (or sun's point} theorem)

The sun is a huge point light source, which is a mixed light luminous source integrated by countless luminous points (small point light sources). Each light-emitting point emits innumerable linear rays according to any spatial angle, and different light-emitting points can emit light of the same spatial angle and different spatial angles. Therefore, in the group of sunlight mixed rays, there are countless parallel rays and countless cross rays with different spatial angles, and the cross rays intersect at one point to form an intersection. So, sunlight is made up of parallel rays and intersecting rays. Outside the sun light source, the sun rays passing through any direction are always parallel rays; the sun rays passing through any point are cross rays. The duality of the horizontal intersection of the sun's rays (or the theorem of the sun's point orientation) is defined according to the spatial geometric angles of the sun's rays corresponding to each other, and is relative. When the crossed sun rays pass through the prism, the light color and spectrum are formed after repeated and orderly reflection of the light channel.

1.3.7 The principle of light color generated by light color entrance and prism 
The sunlight (or white light) produces seven light colors after passing through the prism, and the effective incident area that produces the light color effect on the incident surface is called the light color entrance. When seven adjacent white light chains (light particle chain lines) enter the seven interstitial channels between the eight glass molecules juxtaposed along the side length direction on the triangular prism surface at seven different ordered angles, the order of the light channels is Under the action of reflection, seven light colors (red light, orange light, yellow light, green light, blue light, indigo light, and violet light) are generated respectively. That is, the light color incident width base is 7 light channels. If the light color incident width is defined in terms of frequency, that is, the hertz width of light color incident is $4.3 \sim 7.5 \times 10^{\wedge} 14 \mathrm{~Hz}$ (red light and violet light frequency). The geometrical width of the incident light color will increase proportionally with the increase of the light band width of the outgoing light color. The Hertz width of the incident light color will always remain unchanged, and the Hertz width of the light color at the entrance and the exit port is the same. When the white light body is incident on the triangular prism surface, because the light particles on the edge directly contact the medium and exert force on the medium molecules, the light color effect is only generated on the geometric edge line of the light body cross-section, and the two opposite Red-orange-yellow light and blue-indigo-violet light are generated on the edge line of the strip respectively, and the green light is the color of interproximal cross light. In the white light body, because the force of the light particles is balanced in all directions, the light color effect does not occur. In the seven-color spectrum, red light, yellow light, blue light, and violet light are the four primary light colors, and orange light, green light, and indigo light are the three adjacent light colors (that is, the cross-superimposed light colors between the two light colors). Red light and violet light are superimposed to form pink light. Therefore, the minimum value of the light color incident width base is four light channels.

\subsubsection{Principle of light refraction}

When the light particles pass through the critical surfaces of different media at various angles, they are simultaneously affected by the attractive forces of different media (including the internal field force of the medium) at the critical point, and the resultant force deflects the movement direction of the light particles and forms refraction.

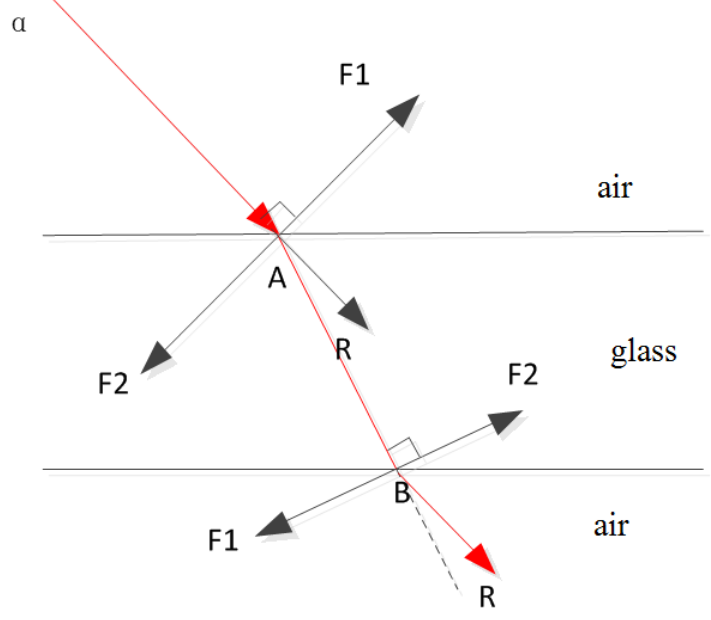

Figure 3: Principle of light refraction

\section{Proof: (Figure 3)}

F1 is the gravitational force of the air on the light particle a, and is perpendicular to the motion direction aA.

F2 is the gravitational force of the glass on the light particle a, and is perpendicular to the motion direction aA.

Because the density of glass is greater than that of air, so: F2>F1

When the light particle a reaches the point A, it is affected by $\mathrm{F} 1$ and $\mathrm{F} 2$ at the same time.

Because: $\mathrm{F} 2>\mathrm{F} 1, \mathrm{AF} 1 \perp \mathrm{aA}, \mathrm{AF} 2 \perp \mathrm{aA}$.

Therefore, the light particle a is deflected by the angle $\mathrm{R}$ in the direction of A F2 to form a refracted light AB.

When the light particle reaches point $\mathrm{B}$, it is affected by $\mathrm{F} 1$ and F2 at the same time.

Because: $\mathrm{F} 2>\mathrm{F} 1, \mathrm{BF} 1 \perp \mathrm{AB}, \mathrm{BF} 2 \perp \mathrm{AB}$

Therefore, the light particle a is deflected by the angle $\mathrm{R}$ in the direction of AF2.

And because: $\mathrm{F} 1=\mathrm{F} 1, \mathrm{~F} 2=\mathrm{F} 2$

Therefore: $\mathrm{R}=\mathrm{R}, \mathrm{Aa} / / \mathrm{Ba}$

Therefore, after the light particle a passes through the flat glass in the air, its motion direction is still parallel.

When the light particle a passes through the critical surface vertically, $\mathrm{F} 1=\mathrm{F} 2$, and its motion direction will not change.

\subsubsection{The theorem of plane reflection of light}

When the linear light passes through the reflection plane, its reflection point on the reflection surface is a fixed contact (belonging to line reflection), the reflected light is still a straight line, and the reflection angle is equal to the incident angle. When the wave-shaped light passes through the reflection plane, its reflection point trajectory on the reflection surface is a contact line segment (belonging to surface reflection), the waveform, wavelength and frequency of the reflected light wave are the same as the incident light wave, the phase is opposite, and the reflection angle is equal to the incident angle. Therefore, the reflected light wave does not suffer from half-wave loss.

\subsubsection{The meaning of light momentum and energy}

According to the principle of relative linear motion of light particles and the energy theorem, it can be deduced that when a light particle moves in a relatively uniform straight line, its momentum is determined by the wavelength, frequency and relative linear velocity, and its energy is determined by the linear velocity (or optical path) decided. Two light waves with the same wavelength, frequency and speed have the same momentum but not necessarily the same energy. The higher the peak of a light wave (the bigger the peak), the more energy 
it contains. Two rays moving in a straight line with uniform velocity have equal momentum and equal energy. The energy contained in the light wave can be increased by means of field force augmentation.

\subsubsection{Light quality duality and light energy theorem (formula)}

The mass of a substance can be defined and measured by the amount and size. The amount of mass is the measure of the material property of the substance, and the size of the mass is the measure of the energy property of the substance. Light particles have both material quality and energy quality. Energy quality is the quality that light particles show in motion to carry energy. Light particles only show material quality when they are in a resting state. The material mass of the light particle is a constant scalar constant, and the energy mass of the light particle is a dynamic vector that changes in motion. When a light particle (including other particles) is accelerated to the speed of light in the field force, its speed stops increasing and becomes constant at the speed of light, but its energy, mass and kinetic energy continue to increase infinitely. The time (or optical path) of the direction of the field force is proportional to the strength of the field force, and shows the energy quality value corresponding to the energy. The calculation formula of the energy of the light particle after reaching the speed of light (or the value after constant speed and stable speed) (that is, the constant speed light energy) is:

Light energy after light speed $\mathrm{E}=$ light particle matter mass $\mathrm{m}$ $\times$ light speed square $\mathrm{C}^{2} \times$ time $\mathrm{t}$

Or: light energy after light speed $\mathrm{E}=$ light particle material mass $\mathrm{m} \times$ light speed square $\mathrm{C} \times$ light path $\mathrm{S}$

Light energy after light speed $\mathrm{E}=$ light particle momentum D $\times$ optical path $\mathrm{S}$

The energy quality of light $\mathrm{M}=$ light energy after the speed of light $\mathrm{E} \div$ the square of the speed of light $\mathrm{C}^{2}=$ light particle matter mass $\mathrm{m} \times$ time $\mathrm{t}$

The light energy $\mathrm{E}=$ the mass of the light particle material $\mathrm{m} \times$ the speed of light square $\mathrm{C}^{2} \times$ the new field time $\mathrm{t} \times$ the new field intensity $\div$ the original field intensity

Total light energy $\mathrm{E}=$ light particle material mass $\mathrm{m} \times$ light speed square $\mathrm{C}^{2} \times$ (original field time + new field time $\times$ new field intensity $\div$ original field intensity)

Variable speed light energy formula: variable speed light energy $\mathrm{E}=$ light particle mass $\mathrm{m} \times$ instantaneous light speed square $\div 2$

The energy quality of sunlight (including other visible light) in nature is exactly equal to the value of material quality, and the increase value is zero, so its energy is at the bottom line of dynamic energy, close to zero, so it will not cause damage to the human body.

\subsubsection{Optical array}

A light array is a connection of light particles that attract each other to form a spatial geometry. It can affect the motion state of light.

\subsubsection{Light quantity and light intensity}

Light quantity refers to the physical quantity of light particles and belongs to the category of energy. Light intensity refers to the number of light particles per unit surface and on it. The intensity of light can be increased by concentrating light.

\subsubsection{Optical wireless power transmission technology}

The principle of laser can be applied to convert a large amount of electrical energy into light energy, and then transfer it to other light energy receiving devices at the speed of light, and then convert it into electrical energy.

\subsection{Important Inferences}

According to the principle of the increase of the motion speed of the light particle after obtaining new kinetic energy in the motion, it is inferred that the motion speed of the light particle will increase infinitely without limit, resulting in superluminal speed. But this state of motion may need to be realized in another gravitational system, such as a black hole. The existence of black holes indicates that there may be another one or more gravitational systems in space, that is, the existence of another one or more universes. Electrolysis of sunlight may generate hydrogen or other elements, revealing the origin of matter in the universe.

\section{Theorem of Heat}

Heat is physical. Heat is the state of energy in which particles of matter undergo thermal motion at various speeds.

Thermal motion is the sum of various linear or curvilinear motions made by particles in the medium. Including rotation, vibration, fluctuation, impact, etc. Particles in thermal motion exhibit various corresponding linear velocities in motion. Each particle that does thermal motion (such as molecule, atom, light particle, etc.) is called a thermon. Among them, light particles are the only source heat particles that carry heat and transfer heat in substances such as air and generate heat. The kinetic energy carried by the hot electrons is called heat. which is:

\section{Heat $=$ thermal mass $\times$ linear velocity squared}

The heat carried by a light particle is a constant (called the photothermal constant, denoted by Y), namely:

Photothermal constant $\mathrm{Y}=$ light constant $\mathrm{Q}=$ mass of light particle $\times$ speed of light squared

The heat carried by a molecule or atom is:

Molecular (or atomic) heat $=$ molecular weight (or atomic weight) $\times$ linear velocity squared

In the process of thermal motion, most of the foreign hot electrons carrying kinetic energy penetrate deep into the vacuum gap between the molecules of the object. Under the 
action of gravity, they change the original state of motion and perform motions such as rotation, vibration, impact, etc., so that the material molecules The movement accelerates, the temperature increases, and the molecular distance increases, so that the solid becomes liquid, the liquid becomes gas, or it explodes and burns.

\section{Photothermal Essence Theory}

Light and heat are both physical and both have mass. Light and heat are the various energy states exhibited by light particles moving at various speeds. The same light particle exhibits both light and heat states in any period of the same motion. For example, sunlight exhibits both light and thermal properties.

Therefore, light is heat, and a certain speed segment of heat is light. Light and heat share basic properties. Light and heat are two states of light particles in the same motion process, in which heat always runs through the whole process of motion. When a beam of light is extinguished, the light particles remain in thermal motion until the energy is exhausted.

After the light particles gain additional heat in motion, their speed increases, and they emit light when they reach the speed of visible light.

The moving light particles act on people's eyes and skin respectively, causing people to send out two biological reactions, which are called light and heat respectively.

\section{References}

[1] Einstein A. On the Electrodynamics of Moving Bodies[J]. Annalen der Physik, 1905, 17.

[2] Master Optical Techniques. ARTHUR S. DEVANY. Translated by Cao Tianning[M]. Beijing: Machinery Industry Press, 2001.

[3] The Development History of Optics. [EB/OL]http://baike.baidu.com/view/4321424.htm\#2

[4] Zhu Ziqiang. Modern Optics Tutorial[M]. Chengdu: Sichuan University Press, 2007.

[5] Xie Jianping. The Foundation of Modern Optics[M]. Beijing: China Science and Technology Press, 2003.

[6] Chen Jiabi. Principles and Applications of Optical Information Science and Technology[M]. Beijing: Higher Education Press, 2004. 